\title{
THE META-ONTOLOGY MODEL OF THE FISH- DISEASE DIAGNOSTIC KNOWLEDGE BASED ON OWL
}

\author{
Yongchang $\mathrm{Shi}^{1}$, Wen $\mathrm{Gao}^{2,3}$, Liang $\mathrm{Hu}^{3}$, Zetian $\mathrm{Fu}^{3, *}$ \\ ${ }^{1}$ Beijing Jiaotong University, No.3 of Shangyuan Residence Haidian Disrict in Beijing, \\ P.R.CHINA 100044 \\ ${ }^{2}$ ShanDong Institute of Business and Technology, 191 BinHai Road Yantai, P.R.CHINA \\ 264005 \\ ${ }^{3}$ College of Engineering, China Agricultural University, No.17 Qinghua Dong Lu, Haidian, \\ Beijing 100083, P.R.CHINA \\ * Corresponding author, Address: College of Engineering, China Agricultural University, \\ No.17 Qinghua Dong Lu, Haidian, Beijing 100083, P.R.CHINA, Tel: +86-010-62736717, \\ Fax:+86-010-62736717,Email: gaowen_sd@163.com
}

Abstract: For improving available and reusable of knowledge in fish disease diagnosis (FDD) domain and facilitating knowledge acquisition, an ontology model of FDD knowledge was developed based on owl according to FDD knowledge model. It includes terminology of terms in FDD knowledge and hierarchies of their class.

Keywords: Ontology; Fish Disease Diagnosis; OWL; knowledge base;

\section{INTRODUCTION}

Aquatic products are an important source of complementary food for improving people's living in China. Since many kinds of fishes have been affected by disease in deeply degree in the north of China, the knowledge engineer in earlier time tried to develop an expert system technology of the fish disease diagnosis in AI.

The designs of intelligent systems for medical diagnosis have been one of the most prolific areas from the very beginnings of AI. The experts in this

Please use the following format when citing this chapter:

Shi, Y., Gao, W., Hu, L. and Fu, Z., 2009, in IFIP International Federation for Information Processing, Volume 294, Computer and Computing Technologies in Agriculture II, Volume 2, eds. D. Li, Z. Chunjiang, (Boston: Springer), pp. 1415-1422. 
area were considered as pioneers in the use of the deep causal models. The systems rapidly showed the advantages of modeling domain knowledge in this way, in contrast with the classical rule-based systems (Palma and Marin 2002). Recently the researches in this area have been paid increasingly attention to the use of ontology models, especially if they are considered as integrated in knowledge based Diagnosis (KBD) techniques, which have proved their efficiency in the design of intelligent diagnosis systems.

Ontology aims at capturing domain knowledge in a generic way and provides a commonly agreed understanding of a domain, which may be reused and shared across applications. and groups(Chandrasekaran, Josephson et al. 1999). Ontologies provide a common vocabulary of an area and define -with different levels of formality- the meaning of the terms and the relations between them. Ontologies are usually organized in taxonomies and typically contain modeling primitives such as classes, relations, functions, axioms and instances(Gruber 1993). Popular applications of ontology include knowledge management, natural language generation, enterprise modeling, knowledge-based systems, ontology-based brokers, and interoperability between systems. (Perez and Benjamins 1999)

The structure of the paper is as follows: the related work about Ontology and OWL in section 2, FDD Knowledge Management Framework is presented in section 3, the formalized expression of FDD Case Knowledge metadata frame using OWL was given in section 4 , and we finally provide conclusions in section 5 .

\section{RELATED WORK}

\section{$2.1 \quad$ Ontology}

In the past, ontological issues were investigated in such areas of AI as theoretical knowledge representation and natural language understanding. Recently, ontological issues are being widely used for the purposes of knowledge sharing and reuse, and object-oriented database design. Ontology can also be seen as the study of the organization and classification of knowledge. Ontological engineering in AI has the practical goal of constructing frameworks for "knowledge" that allow computational systems to tackle knowledge-intensive problems such as natural language processing and real-world reasoning. (Chen and Chan)

The ontology of the domain is not a goal in itself often. Developing the ontology is akin to defining a set of data and their structure for other programs to use. Problem-solving methods, domain-independent 
applications, and software agents use the ontology and knowledge bases which based on the ontology as data. (Chen YanHong 2004)

\section{$2.2 \quad$ OWL}

OWL is a new ontology language for the Semantic Web developed by the World Wide Web Consortium (W3C)Web Ontology Working Group. OWL was primarily designed to represent information about categories of objects and how objects are interrelated the sort of information that is often called the ontology. OWL can also represent information about the objects themselves - the sort of information that is often thought as data.

\section{FDD KNOWLEDGE MANAGEMENT FRAMEWORK}

Ontology design is primarily a categorization process. Good categorizations can facilitate information retrieval. Studies on categorization that pertain to ontology design in the AI field Since the domain ontology of a knowledge-based system is an explicit specification of the objects, concepts, and other entities that are presumed to exist in some area of interest as well as the relationships that are held among them (Gruber 1993), it defines the set of terms and relations of a domain independent of any problem-solving method. Normally, such method-specific formulation of domain knowledge is difficult to reuse in a different application. Therefore, to separate the potentially reusable domain knowledge from the method-specific knowledge is a consideration that guided our structure of the domain ontology. (Chen and Chan)

\section{1 $\quad$ Fish Disease Diagnostic Model}

The fish disease diagnostic question concerning multi-elements is complex. According to our investigation, we identify three broad elements as disease, cause and manifestation, omit others. We specify the relations among the three elements as the category of fish disease diagnostic knowledge. Fig 1 shows the three broad elements and reflects relation among them.

Arrow from node of cause and node of disease reflects causality between cause and disease. Arrow from node of disease to node of manifestation also reflects similar causality between disease and manifestation. The arrow from cause to manifestation reflects pathology. 


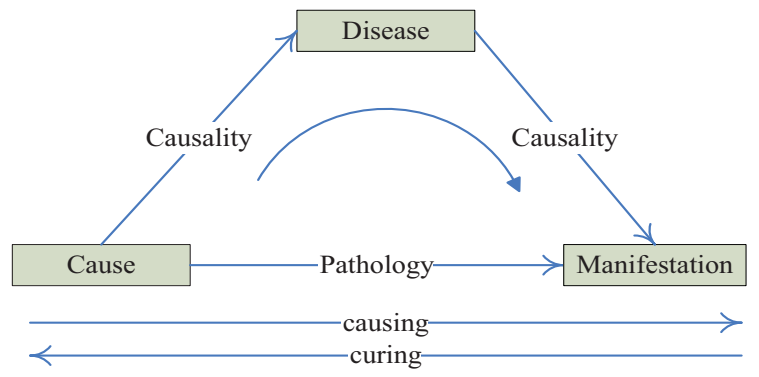

Fig 1 Conceptual Model of FDD

\subsection{FDD Meta-Knowledge Categorizing}

Knowledge acquisition is an important step in developing a knowledge-based system. The knowledge engineer acquires knowledge from one or more application experts who can explain the problem domain. In this process, detailed information on procedural problem-solving such as input and output, domain knowledge, and the entities and relations in that domain are obtained.(Chen and Chan)

We collect and construct the knowledge base for Fish Disease Diagnosis Acquisition System (FDDS). For doing this, we should make use of existed materials and knowledge elicitation conducted primarily through face-to-face interviews and textual cases provided by application experts. In addition, the knowledge engineer learned about the domain from previously published reports, related projects, and a commercial database on disease diagnostic case knowledge management. The strategy adopted for knowledge acquisition (KA) was based on teaching-learning and teaching-back. Teaching learning was used to obtain the knowledge from the experts including verbal data and references. Then, after the knowledge organized, teaching-back was used when the experts validated and clarified the knowledge presented to them by the knowledge engineer.

All this FDD case knowledge is collected and analyzed before stored in the knowledge database. We collect all case knowledge of FDD about ten species of the fish raised in the countryside of northern China.

\subsubsection{FDD Knowledge Base Structure}

In FDD system, the fish disease diagnostic knowledge was organized by knowledge representing scheme. There are two schemes: rule-based scheme and case-based scheme. So knowledge base consists of two parts, rule knowledge base and case knowledge base. They store rule knowledge and case knowledge respectively. Rule knowledge base store knowledge as ifthen schema, and case knowledge base store knowledge as frame schema. For simplification, we only show the case base structure of our FDD model in Fig 2.

The case base is constituted by four parts: the new case base, the cleaned case base, the classified case base and the case knowledge base. 


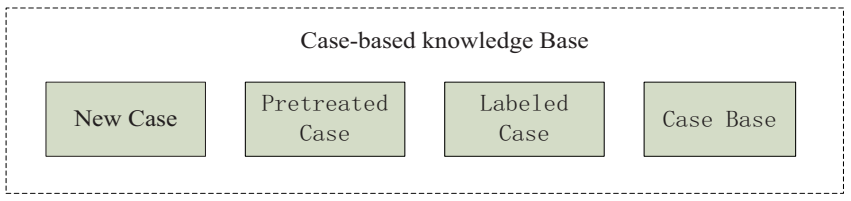

Fig 2 Structure of Case Base

\subsubsection{Categorizing of FDD Case Knowledge Metadata}

In terms of fish disease diagnosis model, there are two class sets which are nominal class subset, metadata for describing case knowledge and metadata for managing case knowledge in fish disease diagnostic case base. The FDD case knowledge class is named as case FDDK in our system.

Table 1 Class in fish disease diagnosis ontology

\begin{tabular}{ll}
\hline \multicolumn{1}{c}{ Class } & $1^{\text {st }} 2^{\text {nd }} 3^{\text {rd }}$ Subclass \\
\hline FDD Case knowledge & New case \\
& Labeled case \\
& Classified case \\
& Case knowledge \\
\cline { 2 - 2 } Role (User) & Knowledge engineer \\
& Domain expert \\
& Manager of knowledge base \\
& Other users \\
& Structure of fish body \\
& Surface of fish body \\
& Head \\
& Body \\
& Tail \\
& Fin \\
& Internal organ \\
& Heart \\
$\ldots$ \\
Species \\
grass carp \\
carp \\
$\ldots$ \\
Growth Phase \\
young fry \\
luce \\
ripe fish \\
Size \\
$\ldots$ \\
\hline
\end{tabular}


Water body

Feed program

Disease

Cause

\subsubsection{Meta-Knowledge Frame of FDD}

The metadata frame of our FDDK is shown in Fig 3 which shows the relationship of different sets in knowledge base.

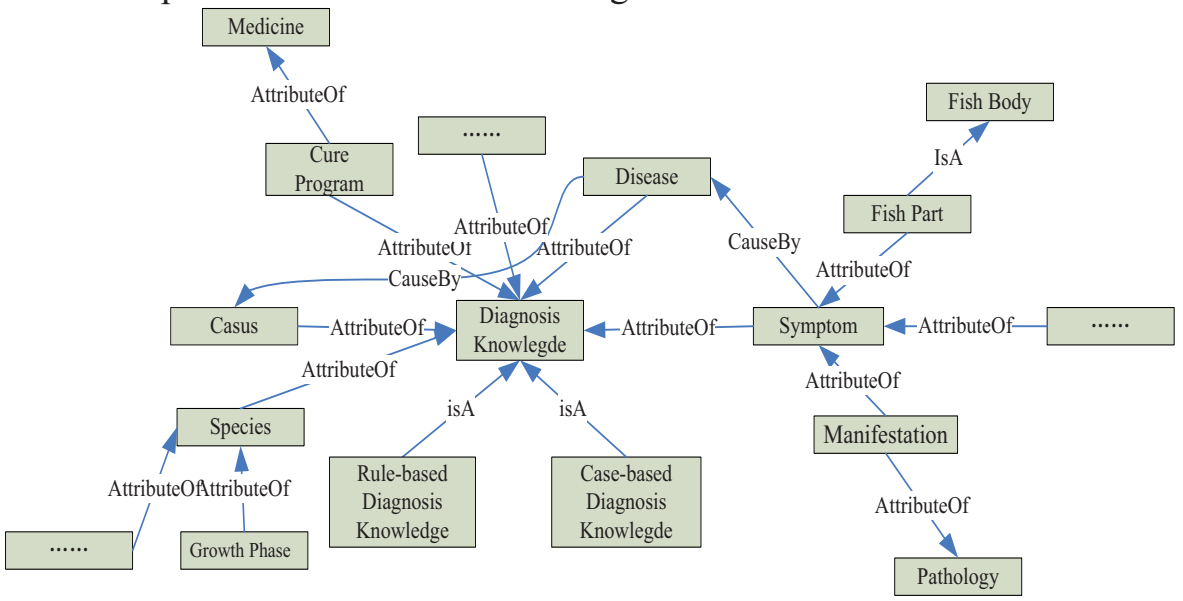

Fig 3 Metadata frame of FDD Knowledge

\section{FORMALIZED FDD META-KNOWLEDGE FRAME BY USING OWL}

OWL (Web Ontology Language) is designed to process the content of information instead of just presenting information to human. OWL can be used to explicitly represent the meaning of terms in vocabularies and the relationships between those terms. This representation and their interrelationships are called Ontology. OWL has more facilities for expressing meaning and semantics than XML, RDF, and RDFS, and thus OWL goes beyond these languages in its ability to represent machine interpretable content on the Web. OWL is a revision of the DAML+OIL web ontology language incorporating lessons learned from the design and application of DAML+OIL.

We give the formalize expression of FDD case knowledge metadata ontology. The formalized process makes the metadata easier to understand by computer. First we give the namespace by defining some entity and then start OWL ontology. We also import ontology; it will add what we have described into the whole knowledge base. 
$<$ ?xml version="1.0"?>

$<$ !DOCTYPE rdf:RDF [

$<$ !ENTITY owl "http://www.w3.org/2002/07/owl\#" >

$<$ !ENTITY xsd "http://www.w3.org/2001/XMLSchema\#" >

$<$ !ENTITY rdfs "http://www.w3.org/2000/01/rdf-schema\#" >

$<$ !ENTITY rdf "http://www.w3.org/1999/02/22-rdf-syntax-ns\#" >

]>

$<$ rdf:RDF xmlns="http://www.owl-

ontologies.com/Ontology1176772345.owl\#"

xml:base="http://www.owl-ontologies.com/Ontology1176772345.owl"

xmlns:xsd="http://www.w3.org/2001/XMLSchema\#"

xmlns:rdfs="http://www.w3.org/2000/01/rdf-schema\#"

xmlns:rdf="http://www.w3.org/1999/02/22-rdf-syntax-ns\#"

xmlns:owl="http://www.w3.org/2002/07/owl\#">

Second we name all class of the system: FDD Knowledge class and subclass of FDD Knowledge, Case Knowledge Class, Role User, Fish class And sub-classes of fish Class and so on, then we define attribute of each class as below.

$<$ owl:Ontology rdf:about="FDDK"/>

$<$ Bacteria rdf:ID="AeromonasPunctata0"/ $>$

$<$ owl:Class rdf:ID="Bacteria" $>$

$<$ rdfs:subClassOf rdf:resource="\#Cause" $/>$

$<$ owl:Class $>$

$<$ PathologyDisplay rdf:ID="black" $>$

$<$ rdfs:comment

rdf:datatype="\&xsd;string" $>\& \# 20307 ; \& \# 33394 ; \& \# 21457 ; \& \# 40657 ;</$ rdfs:commen t>

$<$ /PathologyDisplay $>$

$<$ PathologyDisplay rdf:ID="blood" $>$

$<$ rdfs:comment

rdf:datatype="\&xsd;string" $>\& \# 20986 ; \& \# 34880 ;</$ rdfs:comment $>$

$<$ PathologyDisplay $>$

$<$ FishSpecies rdf:ID="Carp"/>

$<$ owl:Class rdf:ID="Cause">

$<$ owl:disjointWith rdf:resource="\#Disease" $/>$

$<$ owl:disjointWith rdf:resource="\#Manifestation"/>

$<$ owl:disjointWith rdf:resource="\#PathologyDisplay"/>

$<$ rdfs:comment rdf:datatype="\&xsd;string"

$>$ This is a conceptual specification of diseaseCause. $</$ rdfs:comment $>$

$<$ owl:Class $>$

$<$ owl:ObjectProperty rdf:ID="causeBy" $>$

$<$ owl:inverseOf rdf:resource="\#Result_In"/> 
$</$ owl:ObjectProperty $>$

-.....

$</$ owl:Class $>$

\section{CONCLUSION}

After the domain ontology of FDD Knowledge was constructed, we categorized the knowledge stored in FDD knowledge base. The terms in FDD case knowledge are classified and organized in taxonomies. All defined classes in FDD Knowledge formed the framework of FDD case Knowledge and associating classes formed a class hierarchy. We developed an ontology of FDD Case Knowledge. This ontology can then be used as a basis for some applications in a suite of expert-system tools.

\section{ACKNOWLEDGEMENTS}

This work was supported by VEGNET project (Contract No.CN/ASIAIT\&C/005 (89099)), which was funded by the European Union.

\section{REFERENCES}

Chandrasekaran, B., J. R. Josephson, et al. (1999). What Are Ontologies, and Why Do We Need Them?, IEEE Computer Society: 20-26.

Chen YanHong. Component-based Software Reuse of the research and application [D],BeiJing: Capital University of Ecnomics and Business, 2004.

Chen, L. L. and C. W. Chan "Ontology Construction from Knowledge Acquisition." Proceedings of Pacific Knowledge Acquisition Workshop (PKAW 2000): 11-13.

Gruber, T. R. (1993). "A translation approach to portable ontology specifications." Knowledge Acquisition 5(2): 199-220.

Heijst, G. v., A. T. Schreiber, et al. (1997). Using explicit ontologies in KBS development, Academic Press, Inc. 46: 183-292.

Palma, J. and R. Marin (2002). "Modelling Contextual Meta-Knowledge in Temporal Model Based Diagnosis." Proceedings of ECAI: 407-411.

Perez, A. G. and V. R. Benjamins (1999). "Overview of Knowledge Sharing and Reuse Components: Ontologies and Problem-Solving Methods." IJCAI-99 workshop on Ontologies and Problem-Solving Methods (KRR5), Stockholm, Sweden, August 2: 1999. 